\title{
Burden of Malaria in three Endemic Districts of Bhutan: Road to Malaria Elimination
}

\author{
Vishal Chhetri ${ }^{* 1}$, Tobgyel Dukpa ${ }^{2}$, Rinzin Namgay ${ }^{2}$, Kesang Wangchuk ${ }^{2}$, Hari Prasad Pokhrel ${ }^{3}$ \\ ${ }^{1}$ Gelephu Central Regional Referral Hospital, Ministry of Health, Bhutan \\ ${ }^{2}$ Vector Borne Disease Control Programme, Ministry of Health, Bhutan \\ ${ }^{3}$ Gidakom Hospital, Ministry of Health, Bhutan
}

*Corresponding author: vishal_srmc@yahoo.com

\begin{abstract}
Introduction: Bhutan is considered as a low endemic country for malaria. In the last decade, Bhutan has successfully reduced the number of malaria cases with the support from Global Fund and WHO. Sarpang, Samtse and Samdrup Jongkhar districts located in the Southern foothills of Bhutan records the most cases. Method: This retrospective study was undertaken with the available census record maintained in health centers of the three endemic districts and VDCP. Results: There were 892 confirmed malaria cases in 2009 and 41 cases in 2017 in three endemic districts. The slide positivity rate (SPR) declined from $2.2 \%$ in 2009 to $0.1 \%$ in 2017 . The distribution of disease was significantly higher $(p<0.05)$ amongst the male population with the increasing trend of Plasmodium vivax infection. The study found predominance of $\mathrm{P}$. vivax in Samtse (93\%), and Sarpang (62\%) and Plasmodium falciparum in Samdrup Jongkhar (61\%). The last four years (2014-2017) data showed that maximum cases detected were imported, followed by indigenous and introduced malaria; with 50\%, 44\% and 6\%, respectively. The mortality and morbidity due to malaria has magnificently declined in the last decade. Conclusion: The large-scale implementation of vector control interventions, such as insecticide-treated bed nets, indoor residual spraying, free health care service and active vector surveillance has achieved a marked reduction in malaria incidence. Cross border malaria is still a huge challenge for elimination of malaria in Bhutan. Thus, imported malaria is an increasing problem due to high receptivity, and vulnerability.
\end{abstract}

Keywords: Bhutan, Elimination, Malaria, Plasmodium falciparum, Plasmodium vivax

\section{Introduction}

Malaria is a parasitic infection caused by five different Plasmodium species in human, which is transmitted via blood meal of infected female Anopheles mosquitoes. Globally there are at least 300-500 million clinical cases of malaria reported annually ${ }^{[1]}$ and climate change is yet another factor contributing to the malaria menace. ${ }^{[2,3]}$ In the South Asian region, Plasmodium vivax and Plasmodium falciparum are the predominant species causing human malaria and the situation remains the same in Bhutan. ${ }^{[4]}$ In Asia, 19 countries are considered malaria endemic and $62 \%$ of the total population in those countries are at the risk of malaria infection. Yet, remarkably Sri Lanka was declared malaria-free on 5 September, 2016 by the World Health Organization (WHO) ${ }^{[5]}$

Bhutan started to fight against malaria with the support from global fund for AIDS, Tuberculosis and Malaria (GFATM) and WHO. Initially, the program was launched in 1964 as National Malaria Eradication Programme. ${ }^{[6]}$ The vector surveillance system was in its embryonic stage with few health center reporting cases. Later, vector control intervention started with indoor residual spray (IRS) with Dichloro-diphenyl-trichloro-ethane (DDT). The impact of IRS was rapidly apparent, reducing the annual parasite index (API) from 5.1 per 1000 population in 1965 to 2.6 in $1967 .{ }^{[6]}$ Beginning 1971 malaria cases rapidly escalated due to increase in surveillance system and access to passive case detection as population became more aware and accessible to health care facility. The program was renamed to National Malaria Control Programme (NMCP) in 1971. Approximately, 8000 cases were reported each year from 1975 onwards with highest number reported in $1994(39,852) \cdot{ }^{[6]}$ During this period, DDT resistance and drug resistance of P. falciparum strains to chloroquine and sulfadoxine-pyrimethamine were also noted. ${ }^{[7]}$ Interestingly, a recent study by Wangchuk, Drukpa ${ }^{[8]}$ stated there is no evidence of chloroquine-resistant P. vivax in Bhutan. The IRS vector control is done with synthetic pyrethroid, such as deltamethrin and cyfluthrin. ${ }^{[9]}$ The population living in malaria risk area were advised to use insecticide treated bed nets $(\mathrm{ITBN}){ }^{[3]}$ In 2003, the NMCP was renamed to Vector-borne Disease Control Programme (VDCP) extending its activity over other vector diseases and by then Bhutan stepped into Malaria elimination phase.

The vectors suspected for malaria transmission in Bhutan are Anopheles pseudowillmori, An. culicifacies, An. minimus, and An. fluvialitis and these species are relatively abundant during the peak transmission season. ${ }^{[1,2,10]}$ Bhutan has been a low endemic country for malaria and most of the cases are recorded in the Southern districts. Since 1990s, Bhutan has made major progress towards malaria control resulting in pre-elimination and now in the elimination phase, through integrated vector control activities and 


\section{International Journal of Innovative Research in Medical Science (IJIRMS) Volume 04 Issue 01 Jan 2019, ISSN: 2455-8737, Imp. Factor - 4.102 \\ Available online at $-\underline{w w w . i j i r m s . i n}$}

active vector surveillance. ${ }^{[2]}$ Elimination is defined by the WHO as interruption of local transmission to zero indigenous cases of malaria parasite species in a defined geographic area. ${ }^{[11]}$ Despite the reduction of malaria cases in Bhutan, population living along the southern belt are at higher risk of being infected with malaria parasite due to high receptivity and vulnerability. Therefore, the objective of this paper is to present the overview of the malaria situation in the three malaria endemic districts of Bhutan, highlight the progress made by VDCP in controlling and eliminating malaria to achieve National goal 'Bhutan free of indigenous Malaria'.

\section{Material and Methods}

This study was a routine to research retrospective survey. The survey used the data of health centers under Samtse, Sarpang and Samdrup Jongkhar districts from January 2009 to December 2017. All the patients visiting malaria unit with fever were screened for malaria parasites. Patients' personal information recorded in laboratory register were anonymized for the study. Fingertip or venous blood sample were collected and both the thick, and thin blood film were prepared on clean glass slide according to the standard operating procedure (SOP). Fresh Giemsa stain solution was prepared and stained following SOP. Examination of Giemsastained blood films was performed by experienced microscopist, with at least 200 microscopic fields for thin blood films, and at least 200 leukocytes for thick blood films. ${ }^{[9]}$
The entire malaria positive diagnosed either by Microscopy and/or rapid diagnostic test (RDT) were included for the study. The cases were segregated either as $\mathrm{N} 1, \mathrm{~N} 2 / \mathrm{N} 33^{[9]}$ based on their Nationality (N1: Bhutanese, N2\&N3: Non Bhutanese residing in Bhutan \& Non Bhutanese not residing in Bhutan). Furthermore, case classification was done based on foci investigation, and other epidemiological and entomological findings classified as either indigenous, imported, introduced or re-introduced malaria. The data were collected from the VDCP information unit (Epi-info, CDC, USA 2006 database) and validated with the District Malaria supervisor (DMS) and health center based malaria record sheet. Data entry was done using Microsoft excel and analyzed using SPSS. Descriptive information are presented as frequencies, means and percentages. $\mathrm{T}$, and $\mathrm{Z}$ test were used to assess the statistical association considering a p-value of $<0.05$ as statistically significant.

\section{Results and Discussion}

The present retrospective study was undertaken to better understand malaria disease burden in the three endemic districts of Bhutan. In last decade, Bhutan has successfully reduced the malaria cases from 39,852 cases in 1994 to just 62 cases in $2017^{[9]}$ moving towards the national goal of malaria elimination in the country in-line with the regional goal of 'Malaria-Free South-East Asia Region by 2030,. ${ }^{[7]}$

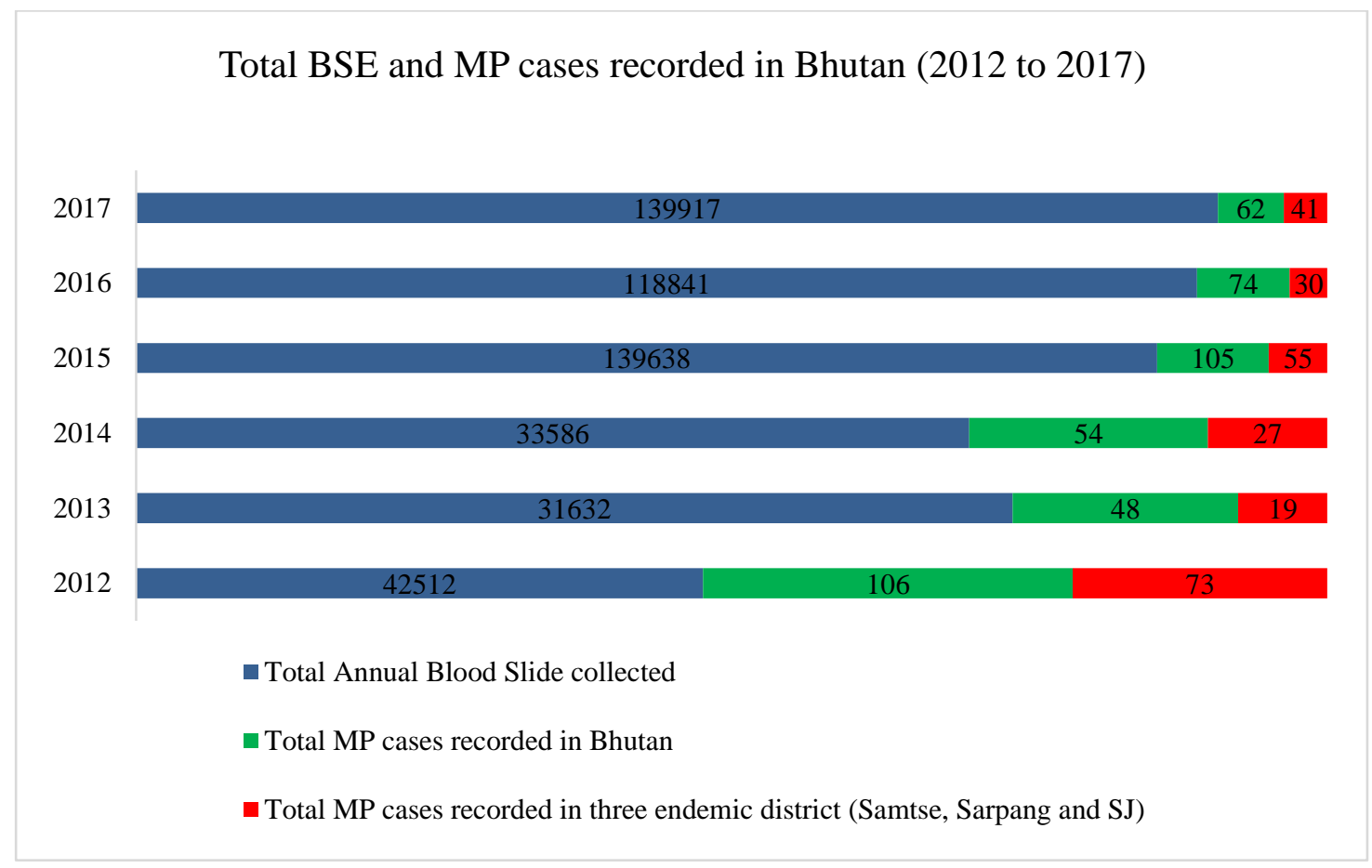

Figure 1: Total Blood slide examined, malaria parasite detected in Bhutan, and in the three endemic districts from 2012-17

A total of 18 death cases due to malaria were recorded from 2007 to 2012 , and one case in 2017 , of which $58 \%$ ( $n=11$ cases) death cases were recorded from Sarpang district. The mortality due to malaria cases has declined to negligible in 2016 from $2.3 \%$ per 100,000 in 2000 (http://www.health.gov.bt). Samtse, Sarpang and Samdrup Jongkhar districts, reported maximum number of malaria cases, significantly higher at $\mathrm{p}<0.05$. In 2009 , the average slide positivity rate (SPR) in these three endemic districts was $2.2 \%$ and this continuously declined to $0.9 \%, 0.7 \%, 0.2 \%$ and $<0.2 \%$ in $2010,2011,2012,2013$, respectively and currently (2017) stands at $0.1 \%$. 


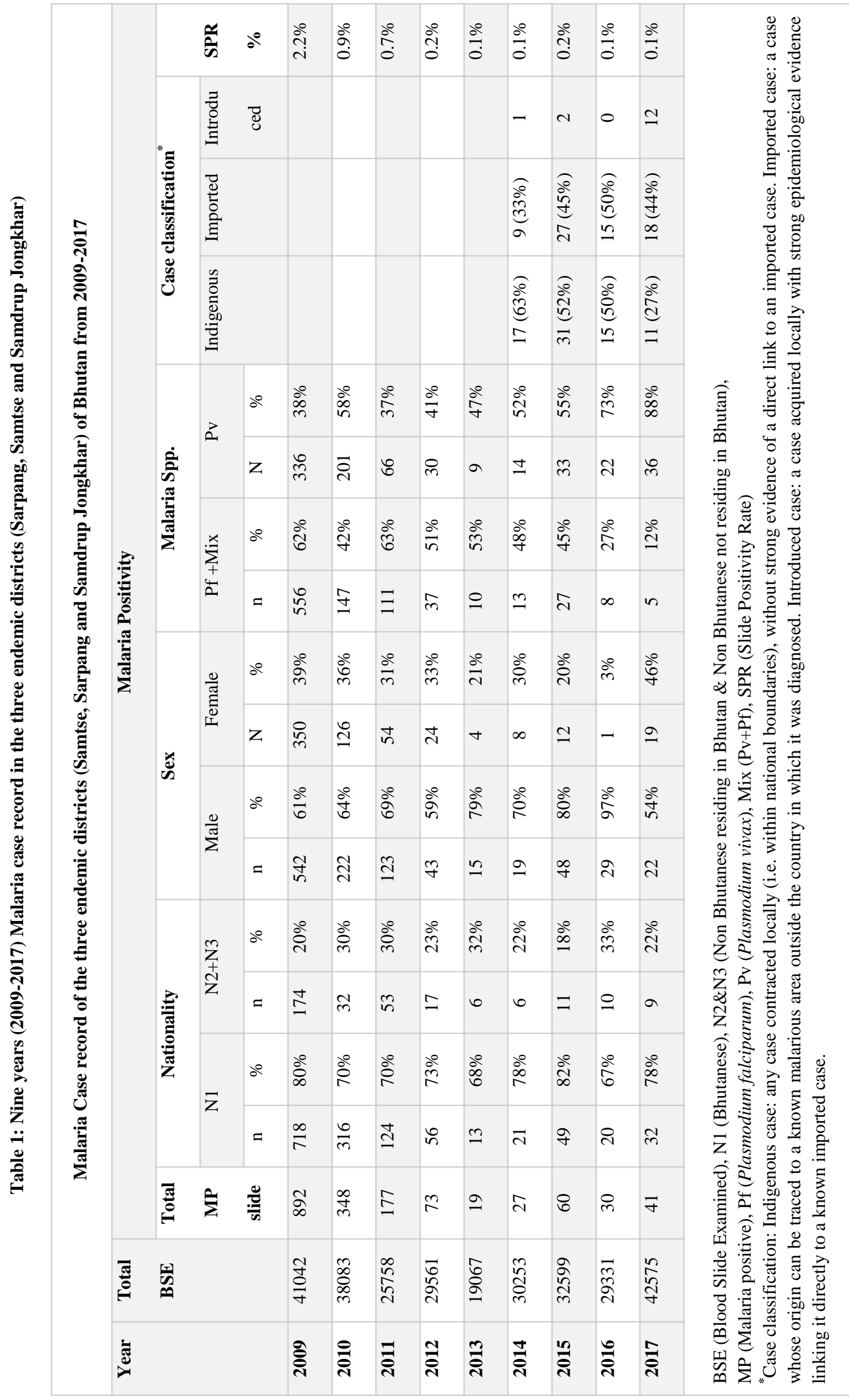




\section{International Journal of Innovative Research in Medical Science (IJIRMS) Volume 04 Issue 01 Jan 2019, ISSN: 2455-8737, Imp. Factor - 4.102 \\ Available online at $-\underline{w w w . i j i r m s . i n}$}

The gender distribution of disease was found significantly higher $(\mathrm{p}<0.05)$ in male population then in female and the infected population were mostly farmers. ${ }^{[12]}$ This could be probably because the paddy fields and irrigation channels located in the wetland are optimal breeding habitats for all vectors. ${ }^{[12]}$ Though P. falciparum was major parasite species until 2013, P. vivax has emerged as dominating species after 2014. This incidence shows the true burden of P. vivax malaria infections ${ }^{[13]}$ and this increasing trend of P. vivax is similar to other South Asian country. ${ }^{[14]}$ Importantly,

A.

Malaria case classification detected from Samtse,Sarpang and S/Jongkhar

$100 \%$

$90 \%$

$80 \%$

$70 \%$

$60 \%$

$50 \%$

$40 \%$

$30 \%$

$20 \%$

$10 \%$

$0 \%$

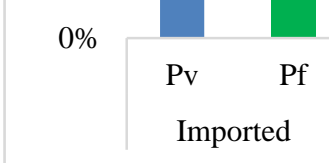

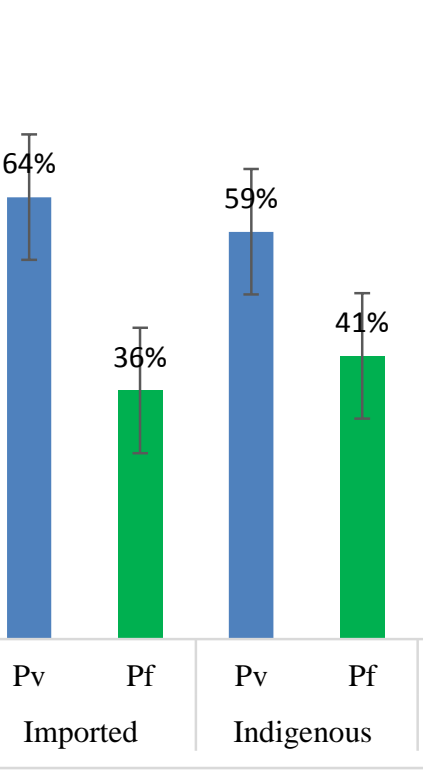

P. vivax exhibits dormant liver stages, which is the main characteristic for emergence of gametocytes before the onset of clinical symptoms and highly prone to resurgence and development of drug resistance. ${ }^{[2,15]}$ Fortunately, the recent study by Wangchuk, Drukpa ${ }^{[8]}$ reported that the P. vivax found in Bhutan can still be treated with chloroquine and primaquine. Moreover, there was no notable difference between the P. vivax found in Bhutanese or nonBhutanese isolates in the neighbor-joining tree.

\section{B.}

Percentage distribution of Malaria parasite species in three endemic districts

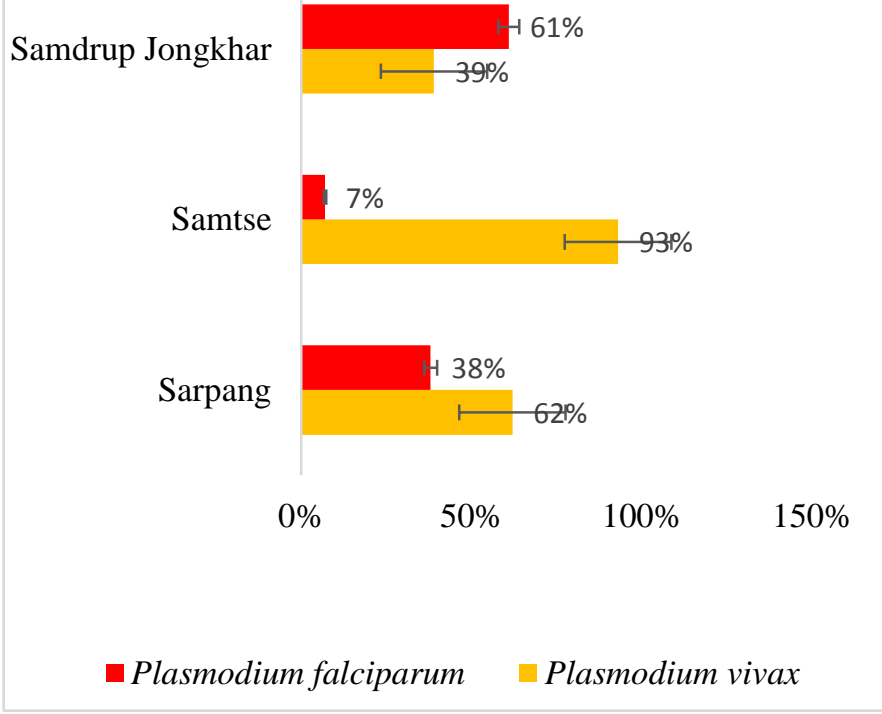

Figure 2: Four consecutive year (2014 to 2017) malaria case detected in the three endemic districts of Bhutan. Fig. A) Percent of Malaria case classification Fig. B) Percent of different malaria species distribution.

The total number of malaria cases detected in Bhutan also includes the foreigners who have entered Bhutan either for a short duration (N2) or stayed for longer (N3), and further based on patient source of infection, epidemiological linkage and travel history, it is classified either as indigenous, imported, induced, introduced or relapse case. ${ }^{[16]}$ In the past four years (2014-2017), the maximum case detected were imported cases, followed by indigenous and introduced malaria with $50 \%, 44 \%$ and $6 \%$, respectively in the three endemic districts. In contrast to the previous study by Yangzom et al., (2012) which reported the dominance of imported P. falciparum in Bhutan, the current study shows P. vivax (64\%) as the predominant imported species. The number of imported malaria positive includes active case detection at the mega-project sites. All malaria cases are treated with the anti-malarial drug therapy, as per the National Guidelines for management of malaria in Bhutan, 2014. ${ }^{[2,4]}$ The imported cases might transmit parasite to community if the vectors are present thus, imported malaria is an increasing problem in many countries ${ }^{[17]}$ and a threat to malaria elimination. In Bhutan there is no evidence of malaria case transmitted either by blood transfusion or congenital. ${ }^{[18,19]}$

The past four years (2014-2017) data showed the diversity of parasite species in the three endemic districts. Samtse and Sarpang detected the predominance of P. vivax with $93 \%$ and $62 \%$, whereas, Samdrup Jongkhar has seen predominance of $\mathrm{P}$. falciparum $(61 \%)$. This could be due to the cross border transmission, as P. falciparum is the predominant parasite species in Assam that share border to Samdrup Jongkhar and Sarpang. ${ }^{[4]}$ Moreover, every year Sarpang district recorded the majority of indigenous cases which is in congruent with the earlier finding by Yangzom et al., (2012). Sarpang shares its border with the Indian state of Assam where malaria is endemic and contributes to more than $5 \%$ of the total cases recorded in India. In addition, malaria transmission is perennial with both winter vector (An. fluviatilis) and the monsoon species (An. dirus) ${ }^{[4]}$ and is therefore, considered high risk malaria transmission. Nevertheless India is also moving towards malaria elimination by $2027 . .^{[11]}$ 


\section{Average monthly malaria case detection in 3 endemic districts for past 4 consequetive years (2014-2017)}

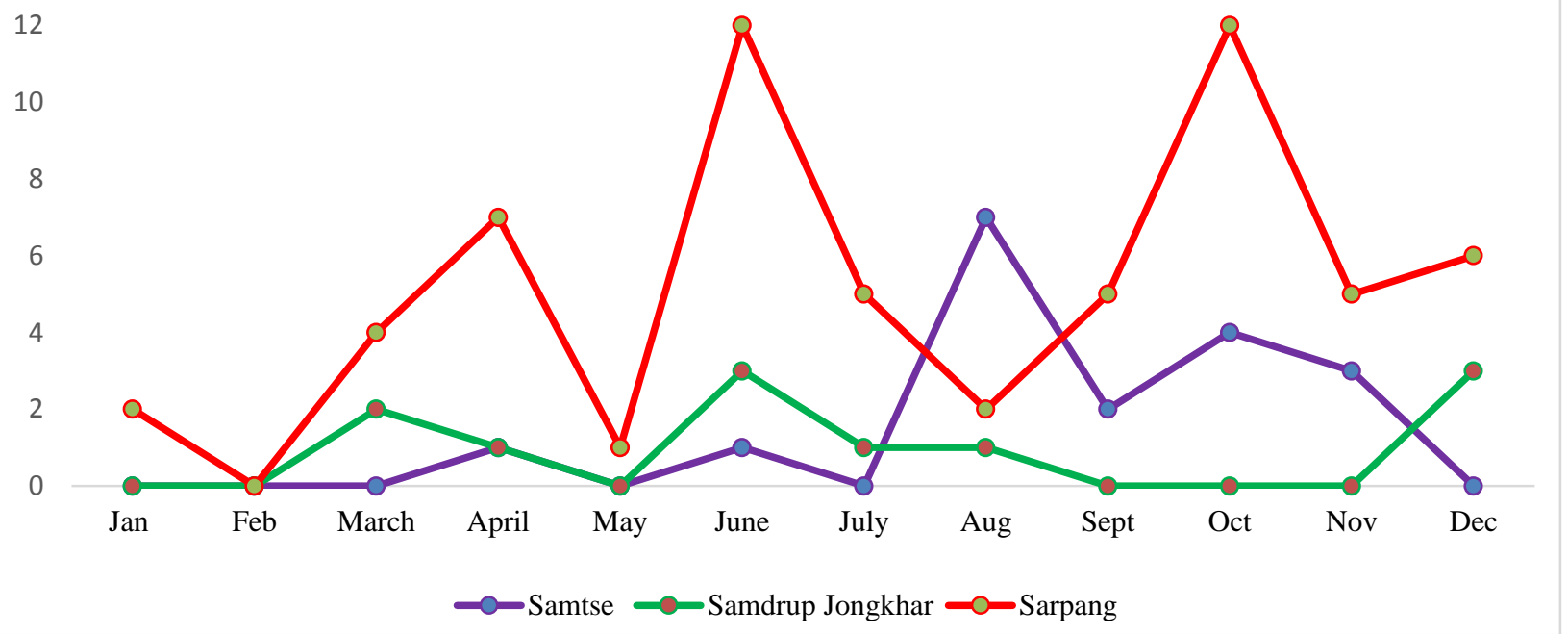

Figure 3: Seasonal distribution of malaria in the three endemic districts of Bhutan. Although the malaria cases were detected throughout the year, the highest number of cases are detected during monsoon season.

The study of seasonal distribution of malaria parasite showed that malaria is detected almost throughout the year but increased during the monsoon, with no notable difference between the two common malaria parasite species. Wangdi et al., (2010) reported that temperature and rainfall were the two predictors to forecast malaria infection, true to which the three endemic district shows increased malaria cases during monsoon. Moreover, the prevalence of malaria vectors in all three endemic districts possess the risk of transmission throughout the year. ${ }^{[1,4]}$ Though the vector control interventions is done with IRS in March and August targeting the early and post monsoon months, as malaria transmission is accelerated during rainy season ${ }^{[15]}$ but the effect is short-lived. ${ }^{[2]}$

Malaria transmission in Bhutan occurs as sporadic epidemic along the Southern districts. The evidence-based strategies implemented by the VDCP with early diagnosis and treatment are prospective root of Bhutan's malaria success. ${ }^{[2]}$ The large-scale implementation of control interventions, such as insecticide-treated bed nets, indoor residual spraying, better information and free health care, free-ofcharge diagnosis and treatment has achieved a marked reduction in malaria incidence. The universal coverage of indoor residual spray (IRS) and usage of long lasting insecticide bed nets (LLIN) for vector control is found effective. A total of 62,538 LLIN was distributed in 2017 covering to almost 89,176 population residing under Sarpang, Samtse and Samdrup Jongkhar (Source: VDCP data, 2018). Both the LLIN and IRS use same group of pyrethroid compound as a blanket cover-up and the coverage of IRS is almost 99\% and the usage of LLIN is $>95 \% .{ }^{[2]}$ Finally the success of IEC (inform, educate and communicate), and advocacy along with the formation of active community action group (CAG) and the healthy and happy Bhutanese population. ${ }^{[4]}$ with improved hygiene and sanitation hereby is a factor, reducing the risk of disease and malaria like fever.

Furthermore, all case investigations were integrated with vector surveillance with focused intervention in the targeted foci, thereby interrupting local transmission of malaria. Active vector surveillance in foci area and active case detection was initiate during elimination phase. Of recent, malaria surveillance system has been integrated with health information and management system (HIMS) and developed customized, and effective surveillance module (District Health Information System-DHIS2), Malaria Tracing System with two tools of malaria case diagnosis, treatment, outcome and case investigation and another to track foci identification being introduced. The bio-assay test on both LLIN and IRS chemical is conducted to study the efficacy of insecticide used. Overall, all of these strategies are stepping stones towards reducing the burden of malaria, and then eliminate malaria. Nonavailability of blood sample for genetic analysis and absence of comprehensive fever records were important limitations in this study.

\section{Challenges}

Though Bhutan has successfully reduced the malaria cases, we are still at risk of malaria infection due to high vulnerability and receptivity. The re-emergence of malaria in previously cleared foci and loss of immunity in the population adds risk to both morbidity and mortality. Moreover, sporadic occurrence of cases in 'hard-toreach' villages makes it difficult to implement vector control measures on time. Most importantly the cross border malaria; due to the dependent on migrant laborer for socio-economic development and the porous border is of high concern. The high malaria transmission across the border has direct impact on the malaria situation in Bhutan as India contributes to almost 61 per cent of malaria cases and 41 per cent of malaria deaths in SEAR countries. ${ }^{[6]}$ The presence of various malaria parasites and vector species, climatic and susceptible human population facilitates malaria transmission in tribal areas of the Eastern and NorthEastern Indian states. ${ }^{[10]}$ that share international border with Bhutan. So there is a greater risk of focal outbreaks due to lack of coordinated vector control operations. ${ }^{[4]}$ Commitment and financial 


\section{International Journal of Innovative Research in Medical Science (IJIRMS) Volume 04 Issue 01 Jan 2019, ISSN: 2455-8737, Imp. Factor - 4.102 \\ Available online at - www.ijirms.in}

support from the government and strategies to control cross border malaria needs to be strengthened to achieve malaria elimination in the region. ${ }^{[20]}$ Limited human resources, false history from the malaria infected patients, increasing $P$. vivax infection and development of drug resistance and, P. vivax highly prone to resurgence especially when control efforts cannot be sustained makes it difficult to contain malaria elimination. ${ }^{[13]}$ Though Bhutan ambitiously targeted 'zero indigenous malaria in 2018' but there were still reported cases of indigenous malaria. Nevertheless, Bhutan still strives to eliminate malaria by 2020 and achieve national goal in line with regional aim Malaria free SEAR 2030.

\section{Conclusion}

Sarpang, Samtse and Samdrup Jongkhar districts despite being located along the porous border of India (Assam, Arunachal Pradesh and West Bengal), where the malaria control activity across the border (tribal area) is minimum, the number of malaria cases had been reduced over the last one decade, from 892 in 2009 to just 41 cases in 2017. Most of the malaria cases are imported, followed by indigenous and introduced cases, 50\%, 44\% and 6\%, respectively, with the predominance of $\mathrm{P}$. vivax $(64 \%)$ mainly among the male population. The cross border malaria still remains a challenge to be resolved. The evidence-based strategies implemented by the VDCP with early diagnosis and treatment are prospective root of Bhutan's malaria success. Though there are population at risk to malaria due to high receptivity and vulnerability, the VDCP ambitiously targets to eliminate malaria; zero indigenous malaria by 2020 ' and contribute towards malaria free South East Asia 2030.

\section{Acknowledgement}

We are thankful to the Entomologist and Epidemiology unit, VDCP, Gelephu and district malaria supervisor of Sarpang, Samtse and Samdrup Jongkhar for support during data collection and verification. We are also thankful to REBH for granting ethical clearance to conduct this study.

\section{Conflict Of Interest}

Authors declare no conflict of interest.

\section{Ethical Clerance}

Ethical clearance for this study was granted by Research Ethics Board of Health (REBH), Ministry of Health, Royal Government of Bhutan via letter no: REBH/Approval/2018/066.

\section{Authors Contribution}

CV Conceived and designed the study. PH assisted in data analysis and interpretation. TD, RN and KW participated and helped in data collection and verification. All authors read and agreed for the submission of this manuscript.

\section{Reference}

[1] Namgay, R., et al., A checklist of the Anopheles mosquito species (Diptera: Culicidae) in Bhutan. Acta tropica, 2018. 188: p. 206-212.
[2] Yangzom, T., et al., Malaria control in Bhutan: case study of a country embarking on elimination. Malaria journal, 2012. 11(1): p. 9.

[3] Tobgay, T., C.E. Torres, and K. Na-Bangchang, Malaria prevention and control in Bhutan: successes and challenges. Acta tropica, 2011. 117(3): p. 225-228.

[4] Dev, V., et al., Physiographic and entomologic risk factors of malaria in Assam, India. The American journal of tropical medicine and hygiene, 2004. 71(4): p. 451456.

[5] Simac, J.N., et al., Malaria elimination in Sri Lanka. Journal of Health Specialties, 2017. 5(2): p. 60.

[6] Organization, W.H., World Malaria Report 2011. Geneva: World Health Organization, 2013.

[7] Organization, W.H., Regional Action Plan 2017-2030. Towards 0. Malaria-Free South-East Asia Region. 2017.

[8] Wangchuk, S., et al., Where chloroquine still works: the genetic make-up and susceptibility of Plasmodium vivax to chloroquine plus primaquine in Bhutan. Malaria journal, 2016. 15(1): p. 277.

[9] Organization, W.H., Climbing towards elimination in Bhutan. 2015.

[10] Sharma, R.K., et al., Malaria situation in India with special reference to tribal areas. The Indian journal of medical research, 2015. 141(5): p. 537.

[11] Narain, J.P. and L.M. Nath, Eliminating malaria in India by 2027: The countdown begins! The Indian journal of medical research, 2018. 148(2): p. 123.

[12] Wangdi, K., et al., Spatio-temporal patterns of malaria infection in Bhutan: a country embarking on malaria elimination. Malaria journal, 2011. 10(1): p. 89.

[13] Group, V.W., Targeting vivax malaria in the Asia Pacific: the Asia Pacific Malaria Elimination Network vivax working group. Malaria journal, 2015. 14(1): p. 484.

[14] Congpuong, K. and R. Ubalee, Population Genetics of Plasmodium vivax in Four High Malaria Endemic Areas in Thailand. The Korean journal of parasitology, 2017. 55(5): p. 465.

[15] Wangdi, K., et al., Development of temporal modelling for forecasting and prediction of malaria infections using time-series and ARIMAX analyses: a case study in endemic districts of Bhutan. Malaria Journal, 2010. 9(1): p. 251.

[16] Organization, W.H., Terminology of malaria and of malaria eradication: report of a drafting committee. 1963.

[17] Kain, K.C., et al., Imported malaria: prospective analysis of problems in diagnosis and management. Clinical infectious diseases, 1998. 27(1): p. 142-149.

[18] Chhetri, V., K. Yangchen, and C. Dawa, Increasing Trend of Clinical Laboratory Testing at Gelephu Central Regional Referral Hospital, Bhutan.

[19] Chhetri, V., Sero-prevalence of Transfusion Transmitted Infections among Blood Donors at CRRH, Gelephu, Bhutan. Journal of Medical Science And clinical Research, 2018. 6(5).

[20] Wangdi, K., et al., Malaria elimination in India and regional implications. The Lancet infectious diseases, 2016. 16(10): p. e214-e224. 